\title{
Effect of interaction with neutrons in matter on flavor conversion of super-light sterile neutrino with active neutrino
}

\author{
Wei Liao, Yuchen Luo and Xiao-Hong Wu \\ Institute of Modern Physics, School of Sciences, \\ East China University of Science and Technology, \\ 130 Meilong Road, Shanghai 200237, P.R. China
}

\begin{abstract}
A super-light sterile neutrino was proposed to explain the absence of the expected upturn of the survival probability of low energy solar boron neutrinos. This is because this super-light sterile neutrino can oscillate efficiently with electron neutrino through a MSW resonance happened in Sun. One may naturally expect that a similar resonance should happen for neutrinos propagating in Earth matter. We study the flavor conversion of this super-light sterile neutrino with active neutrinos in Earth matter. We find that the scenario of the super-light sterile neutrino can easily pass through possible constraints from experiments which can test the Earth matter effect in oscillation of neutrinos. Interestingly, we find that this is because the naively expected resonant conversion disappears or is significantly suppressed due to the presence of a potential $V_{n}$ which arises from neutral current interaction of neutrino with neutrons in matter. In contrast, the neutron number density in the Sun is negligible and the effect of $V_{n}$ is effectively switched off. This enables the MSW resonance in Sun needed in oscillation of the super-light sterile neutrino with solar electron neutrinos. It's interesting to note that it is the different situation in the Sun and in the Earth that makes $V_{n}$ effectively turned off and turned on respectively. This observation makes the scenario of the super-light sterile neutrino quite interesting.
\end{abstract}

Keywords: Beyond Standard Model, Neutrino Physics

ARXIV EPRINT: 1403.2559 


\section{Contents}

1 Introduction $\quad 1$

2 Super-light sterile neutrino and its oscillation in $4 \nu$ and $3 \nu$ formalism 2

3 Energy Levels and the flavor conversion of the super-light sterile neutrino 8

4 Formulation of oscillation of super-light sterile neutrino in the Earth 12

5 Conclusion $\quad 16$

\section{Introduction}

Among many candidates of sterile neutrino proposed in literature, a super-light sterile neutrino appears to be very interesting $[1,2]$. With a mass squared difference with $\nu_{1}$ at around $\Delta m_{01}^{2} \approx(0.5-2) \times 10^{-5} \mathrm{eV}^{2}$ and a mixing with electron neutrino around $\sin ^{2} 2 \theta_{01} \approx 0.001-0.005[2,3]$, this sterile neutrino can help to explain the absence of the upturn of the solar boron neutrino spectrum at energy $E_{\nu} \lesssim 4 \mathrm{MeV}[4-7]$ which is expected in the LMA MSW [8-12] solution of the solar neutrino anomaly. This is achieved with the help of a MSW resonant conversion of this super-light sterile neutrino with solar electron neutrino when neutrino travels from the interior of the Sun to the outside $[1,2]$.

One may naturally expect that there might exist a resonance of flavor conversion between this sterile neutrino and electron neutrino when neutrinos propagate in Earth matter. If a resonance happen, the effective mixing angle between this sterile neutrino and electron neutrino can reach maximal and the oscillation phase would be around $V_{e} L \sin 2 \theta_{01}$ which can be of order one for a long enough oscillation length $L$, e.g. for neutrinos crossing the core of the Earth. Hence, the probability of flavor conversion could be large in resonance region. In particular, this would lead to a suppression of the total flux of active neutrinos when neutrinos pass through the core of the Earth.

Since the mass squared difference $\Delta m_{01}^{2}$ is several to ten times smaller than the solar mass squared difference $\Delta m_{21}^{2}$, the associated resonance may happen at an energy much lower than the energy of the $1-2$ resonance which is around $100-200 \mathrm{MeV}$ in Earth matter. In particular, this resonant conversion of sterile neutrino with electron neutrino may happen for high energy solar neutrinos, supernovae neutrinos and for low energy atmospheric neutrinos. Hence, constraints on this super-light sterile neutrinos may exist in low energy atmospheric neutrino data, supernovae neutrino data and the test of the Earth matter effect in solar neutrino data. In this article (for other works discussing the Earth effect in oscillation of light sterile neutrino with active neutrinos, see e.g. [13, 14]) we examine the oscillation of the super-light sterile neutrino with active neutrinos in the Earth 
matter. Interestingly, we find that the naively expected resonant conversion between this super-light sterile neutrino and active neutrino disappears or is significantly suppressed in the presence of a sizeable potential from the neutral current interaction with matter. This is different from the oscillation happened in the Sun for which the neutron number density is low and the effect from neutral current can be effectively neglected. Consequently, we find that this super-light sterile neutrino passes the possible constraints from experiments testing neutrino oscillation in Earth matter.

In the following of the present article we will first give a brief review of the formalism and the convention of neutrino oscillation with a super-light sterile neutrino. Then we will discuss the reduction of the $4 \nu$ formalism of neutrino oscillation in Earth matter to a $3 \nu$ formalism at energy $<1 \mathrm{GeV}$. In this $3 \nu$ formalism we will discuss in detail, with the help of a baseline dependent average potential, the energy levels of neutrinos and the oscillation of the super-light sterile neutrino with active neutrinos. We will show how the naively expected resonant conversion of this super-light sterile neutrino and active neutrinos disappears in the presence of $V_{n}$, an effective potential coming from neutral current interaction with neutrons in matter. For the completeness of the discussion in this article, we will then discuss the description of the oscillation of super-light sterile neutrino using the baseline dependent average potential and show that the discussion using this baseline dependent potential is indeed a valid and good description despite the fact that Earth matter has complicated density profile. Finally we conclude.

\section{Super-light sterile neutrino and its oscillation in $4 \nu$ and $3 \nu$ formalism}

In the presence of a super-light sterile neutrino, the Hamiltonian governing the oscillation of $\nu=\left(\nu_{s}, \nu_{e}, \nu_{\mu}, \nu_{\tau}\right)^{T}$, the neutrino in flavor base, is

$$
H=U H_{0} U^{\dagger}+V,
$$

where

$$
\begin{aligned}
H_{0} & =\frac{1}{2 E} \operatorname{diag}\left\{\Delta m_{01}^{2}, 0, \Delta m_{21}^{2}, \Delta m_{31}^{2}\right\}, \\
V & =\operatorname{diag}\left\{0, V_{e}+V_{n}, V_{n}, V_{n}\right\} .
\end{aligned}
$$

$V_{e}=\sqrt{2} G_{F} n_{e}$ and $V_{n}=-\frac{1}{\sqrt{2}} G_{F} n_{n}$ where $n_{e}$ and $n_{n}$ are number densities of electron and neutron in matter. $U$ is a $4 \times 4$ unitary matrix describing the mixing of neutrinos. Neglecting CP violating phases, it can be parameterized by

$$
U=R\left(\theta_{23}\right) R\left(\theta_{13}\right) R\left(\theta_{12}\right) R\left(\theta_{02}\right) R\left(\theta_{01}\right) R\left(\theta_{03}\right),
$$

where $R\left(\theta_{i j}\right)$ is a $4 \times 4$ rotation matrix with a mixing angle $\theta_{i j}$ appearing at $i$ and $j$ entries, e.g.

$$
R\left(\theta_{01}\right)=\left(\begin{array}{cccc}
\cos \theta_{01} & \sin \theta_{01} & 0 & 0 \\
-\sin \theta_{01} & \cos \theta_{01} & 0 & 0 \\
0 & 0 & 1 & 0 \\
0 & 0 & 0 & 1
\end{array}\right)
$$


and

$$
R\left(\theta_{02}\right)=\left(\begin{array}{cccc}
\cos \theta_{02} & 0 & \sin \theta_{02} & 0 \\
0 & 1 & 0 & 0 \\
-\sin \theta_{02} & 0 & \cos \theta_{02} & 0 \\
0 & 0 & 0 & 1
\end{array}\right)
$$

$\theta_{12,13,23}$ are mixing angles governing the flavor conversion of solar neutrinos, reactor neutrinos at short baseline and atmospheric neutrinos separately and they have been measured in solar, atmospheric, long baseline and reactor neutrino experiments [15-18]. If $\theta_{0 i}(i=1,2,3)$ are all zero, the mixing matrix $U=R\left(\theta_{23}\right) R\left(\theta_{13}\right) R\left(\theta_{12}\right)$ and it reproduces the PMNS mixing matrix without $\mathrm{CP}$ violating phase for three active neutrinos. For antineutrinos the Hamiltonian is (2.1) with $V$ replaced by $-V$.

As observed in [2], the presence of a super-light sterile neutrino with $\Delta m_{01}^{2} \approx(0.5-$ 2) $\times 10^{-5} \mathrm{eV}^{2}$ and $\sin ^{2} 2 \theta_{01} \approx 0.001-0.005$ can lead to a further suppression of the flux of the solar electron neutrinos at energy around $\lesssim 4 \mathrm{MeV}$. Hence it provides an explanation of the absence of the upturn of the solar neutrino flux at this energy range. For a small but non-zero $\theta_{02}$, similar phenomena would occur for solar electron neutrinos [2].

For a small but non-zero $\theta_{03}$, solar electron neutrinos are basically not affected by it but a resonant $\nu_{s}-\nu_{\tau}\left(\right.$ or $\left.\bar{\nu}_{s}-\bar{\nu}_{\tau}\right)$ oscillation of atmospheric neutrino is expected to happen for energy around $10-15 \mathrm{GeV}$ [2]. So the scenario with a non-zero $\theta_{03}$ can be tested in future atmospheric neutrino experiments and long baseline experiments.

In this article we study the effect of $U_{s 1}$ and $U_{s 2}$ (basically $\sin \theta_{01}$ and $\sin \theta_{02}$ ) in oscillation of neutrinos in Earth matter. For energy as high as $\gtrsim 1 \mathrm{GeV}$, oscillation of active neutrinos with sterile neutrino due to effects of $U_{s 1}$ and $U_{s 2}$ would be strongly suppressed since $\Delta m_{01}^{2} \approx(0.5-2) \times 10^{-5} \mathrm{eV}^{2}$ and $\Delta m_{21}^{2} \approx 7.5 \times 10^{-5} \mathrm{eV}^{2}$ and the oscillation lengths of $1-2$ and $0-1$ oscillation are all much longer than the diameter of the Earth for such high energy. Instead, we concentrate on oscillation of neutrinos for energy $<1 \mathrm{GeV}$. In this energy range, the $2-3$ oscillation is pretty fast and consequently we can reduce the $4 \nu$ formalism to a $3 \nu$ formalism in the study of the resonant oscillation between sterile neutrino and active neutrino. We will see that this $3 \nu$ formalism is more convenient for later discussion.

The $3 \nu$ formalism can be achieved from (2.1) as follows. We can first rotate the Hamiltonian by $R\left(\theta_{23}\right)$ and $R\left(\theta_{13}\right)$. Introducing $\nu^{\prime}=\left(\nu_{s}, \nu_{e}^{\prime}, \nu_{\mu}^{\prime}, \nu_{\tau}^{\prime}\right)^{T}$, which is obtained by

$$
\nu^{\prime}=R\left(\theta_{23}\right) R\left(\theta_{13}\right) \nu,
$$

we find that the Hamiltonian for $\nu^{\prime}$ is

$$
\begin{aligned}
H^{\prime} & =\left(R\left(\theta_{23}\right) R\left(\theta_{13}\right)\right)^{\dagger} H R\left(\theta_{23}\right) R\left(\theta_{13}\right) \\
& =U^{\prime} H_{0}\left(U^{\prime}\right)^{\dagger}+V^{\prime}
\end{aligned}
$$


where $U^{\prime}=R\left(\theta_{12}\right) R\left(\theta_{02}\right) R\left(\theta_{01}\right) R\left(\theta_{03}\right)$ and

$$
V^{\prime}=\left(\begin{array}{cccc}
0 & 0 & 0 & 0 \\
0 & V_{e} \cos ^{2} \theta_{13}+V_{n} & 0 & V_{e} \sin \theta_{13} \cos \theta_{13} \\
0 & 0 & V_{n} & 0 \\
0 & V_{e} \sin \theta_{13} \cos \theta_{13} & 0 & V_{e} \sin ^{2} \theta_{13}+V_{n}
\end{array}\right)
$$

eq. (2.7) can be rewritten as

$$
H^{\prime}=\left(\begin{array}{lc}
\widehat{H}^{\prime} & S \\
S^{\dagger} & \frac{\Delta m_{31}^{2}}{2 E} \cos ^{2} \theta_{03}+\frac{\Delta m_{01}^{2}}{2 E} \sin ^{2} \theta_{03}+V_{e} \sin ^{2} \theta_{13}+V_{n}
\end{array}\right) .
$$

$\widehat{H}^{\prime}$ is a $3 \times 3$ matrix

$$
\widehat{H}^{\prime}=\hat{U} \widehat{H}_{0}^{\prime} \hat{U}^{\dagger}+\widehat{V}^{\prime}
$$

$\widehat{H}_{0}^{\prime}, \widehat{V}^{\prime}$ and $\hat{U}$ are

$$
\begin{aligned}
\widehat{H}_{0}^{\prime} & =\operatorname{diag}\left\{\frac{\Delta m_{01}^{2}}{2 E} \cos ^{2} \theta_{03}+\frac{\Delta m_{31}^{2}}{2 E} \sin ^{2} \theta_{03}, 0, \frac{\Delta m_{21}^{2}}{2 E}\right\}, \\
\widehat{V}^{\prime} & =\operatorname{diag}\left\{0, V_{e} \cos ^{2} \theta_{13}+V_{n}, V_{n}\right\}, \\
\hat{U} & =\hat{R}\left(\theta_{12}\right) \hat{R}\left(\theta_{02}\right) \hat{R}\left(\theta_{01}\right),
\end{aligned}
$$

where $\hat{R}\left(\theta_{i j}\right)$ is a $3 \times 3$ matrix with mixing angle appearing at $i$ and $j$ entries, e.g.

$$
\hat{R}\left(\theta_{01}\right)=\left(\begin{array}{ccc}
\cos \theta_{01} & \sin \theta_{01} & 0 \\
-\sin \theta_{01} & \cos \theta_{01} & 0 \\
0 & 0 & 1
\end{array}\right)
$$

and

$$
\hat{R}\left(\theta_{12}\right)=\left(\begin{array}{ccc}
1 & 0 & 0 \\
0 & \cos \theta_{12} & \sin \theta_{12} \\
0 & -\sin \theta_{12} & \cos \theta_{12}
\end{array}\right)
$$

$S$ is a $3 \times 1$ matrix

$$
S=\hat{U}\left(\begin{array}{c}
\frac{1}{2} \sin 2 \theta_{03}\left(\frac{\Delta m_{31}^{2}-\Delta m_{01}^{2}}{2 E}\right) \\
0 \\
0
\end{array}\right)+\left(\begin{array}{c}
0 \\
\frac{1}{2} \sin 2 \theta_{13} V_{e} \\
0
\end{array}\right),
$$

From (2.6) one can see that $\nu_{e}^{\prime}$ is mainly $\nu_{e}$ and has a small component of $\nu_{\mu}$ and $\nu_{\tau}$. It has a probability $\cos ^{2} \theta_{13}$ being $\nu_{e}$ and a probability $\sin ^{2} \theta_{13}$ being $\nu_{\mu}$ and $\nu_{\tau}$. Disappearance of solar $\nu_{e}$ can be effectively studied by examining the oscillation of $\nu_{e}^{\prime}$ to other neutrinos [2]. 

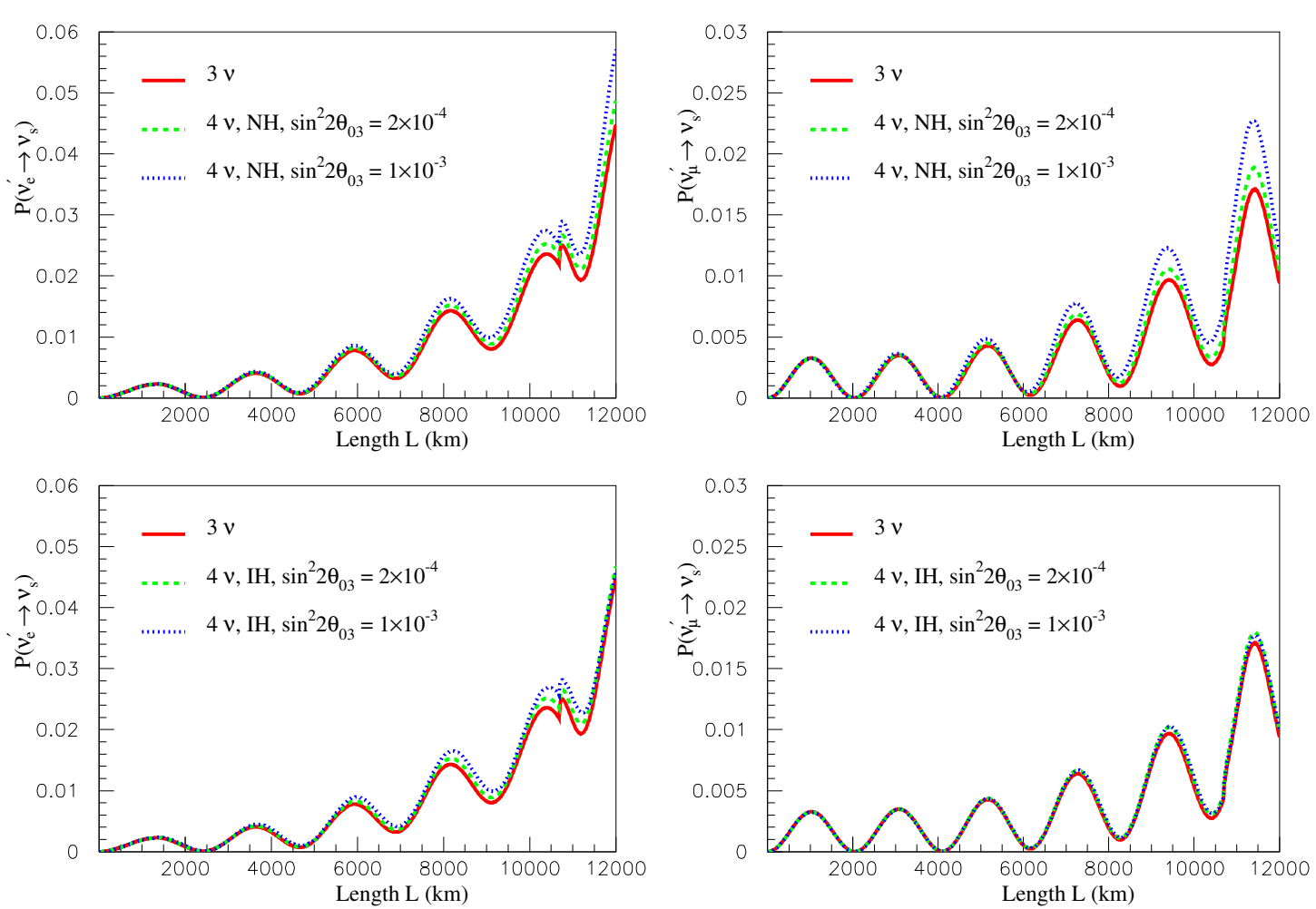

Figure 1. $\nu_{e}^{\prime}-\nu_{s}$ and $\nu_{\mu}^{\prime}-\nu_{s}$ conversion probability in three neutrino framework v.s. in four neutrino framework. $E=60 \mathrm{MeV}, \Delta \widehat{m}_{01}^{2}=0.5 \times 10^{-5} \mathrm{eV}^{2}, \sin ^{2} 2 \theta_{02}=0.005, \sin ^{2} 2 \theta_{01}=0$.

As is well known, $2-3$ resonance happens for energy $5-7 \mathrm{GeV}$ in the Earth and for energy $<1 \mathrm{GeV},\left|\Delta m_{31}^{2} /(2 E)\right| \gg V_{e}$. In the Earth, the neutron number density is roughly of the same order of the electron number density. It is estimated that [19]

$$
R_{n}= \begin{cases}0.024, & \text { mantle } \\ 0.146, & \text { core }\end{cases}
$$

where $R_{n}=\left(n_{n}-n_{e}\right) / n_{e}$. So we can conclude that for energy $<1 \mathrm{GeV}$ we have $\left|\Delta m_{31}^{2} /(2 E)\right| \gg$ $\left|V_{n}\right|$. From this conclusion we can find from eqs. (2.9) and (2.16) that the correction to the probability of the $\nu_{s}$ to $\nu_{e}^{\prime}$ or $\nu_{\mu}^{\prime}$ conversion through $\nu_{\tau}^{\prime}$ and vice versa, i.e.

$$
\nu_{s} \leftrightarrows \nu_{\tau}^{\prime} \leftrightarrows\left(\nu_{e}^{\prime}, \nu_{\mu}^{\prime}\right)
$$

is suppressed by factor

$$
\sin 2 \theta_{03} \frac{2 E V_{e}}{\Delta m_{31}^{2}} \sin 2 \theta_{13} \quad \text { or } \quad \sin ^{2} 2 \theta_{03}
$$

For $\sin ^{2} 2 \theta_{13} \approx 0.09 \quad[16-18]$ and $\sin ^{2} 2 \theta_{03} \lesssim 0.001$, this amplitude is maximally of order $10^{-3}$ for $E<1 \mathrm{GeV}$. Hence the effects of $\nu_{s}-\left(\nu_{e}^{\prime}, \nu_{\mu}^{\prime}\right)$ oscillation through $\nu_{\tau}^{\prime}$ is small and can be neglected.

In figure 1 we give the plots of $\nu_{s}-\left(\nu_{e}^{\prime}, \nu_{\mu}^{\prime}\right)$ conversion versus $L$, the length of neutrino trajectory in the Earth, with matter effect included. The plots in the $4 \nu$ framework are 
calculated using Hamiltonian (2.9) and the plots in the $3 \nu$ framework calculated using $\widehat{H}^{\prime}$ in Hamiltonian (2.9). The horizontal axis $L$ extends to $12000 \mathrm{~km}$ which is about the diameter of the Earth. Relevant neutrino parameters in our calculation are [15]

$$
\begin{array}{rlrl}
\Delta m_{21}^{2} & =(7.50 \pm 0.20) \times 10^{-5} \mathrm{eV}^{2}, \quad\left|\Delta m_{32}^{2}\right| & =\left(2.32_{-0.08}^{+0.12}\right) \times 10^{-3} \mathrm{eV}^{2}, \\
\sin ^{2} 2 \theta_{12} & =0.857 \pm 0.024, & \sin ^{2} 2 \theta_{23} & >0.95 .
\end{array}
$$

We use $\sin ^{2} 2 \theta_{23}=1$ in our calculation. For $\theta_{13}$, we use the central value of a precise measurement achieved by Daya-Bay collaboration [18]:

$$
\sin ^{2} 2 \theta_{13}=0.089 \pm 0.010 \pm 0.005
$$

For other parameters we use the central values in eqs. (2.20), (2.21). Results in figure 1 have been shown both for the case of normal hierarchy $(\mathrm{NH})$ and for the case of inverted hierarchy $(\mathrm{IH})$.

In figure 1 we can see that the result calculated in $3 \nu$ framework agrees with that calculated in $4 \nu$ framework in the case that $\sin ^{2} 2 \theta_{03}$ is small. Actually, for $\theta_{03}=0$ two lines for $4 \nu$ and $3 \nu$ overlap. We see that a non-zero but small $\theta_{03}$ can not change the conversion qualitatively. This agrees with our discussion presented above.

In the following we will discuss effects of $\theta_{01}$ and $\theta_{02}$ in neutrino oscillation and will set $\theta_{03}=0$. So we can study the oscillation of $\nu_{s}-\left(\nu_{e}^{\prime}, \nu_{\mu}^{\prime}\right)$ effectively in a $3 \nu$ framework

$$
i \frac{d}{d t} \widehat{\nu}^{\prime}=\widehat{H} \widehat{\nu}^{\prime}
$$

where $\widehat{\nu}^{\prime}=\left(\nu_{s}, \nu_{e}^{\prime}, \nu_{\mu}^{\prime}\right)^{T} . \widehat{H}$ is given by

$$
\widehat{H}=\hat{U} \widehat{H}_{0} \hat{U}^{\dagger}+\widehat{V}
$$

with $\widehat{H}_{0}$ obtained from $(2.11)$ as

$$
\widehat{H}_{0}=\operatorname{diag}\left\{\frac{\Delta \widehat{m}_{01}^{2}}{2 E}, 0, \frac{\Delta m_{21}^{2}}{2 E}\right\}
$$

where

$$
\Delta \widehat{m}_{01}^{2}=\Delta m_{01}^{2} \cos ^{2} \theta_{03}+\Delta m_{31}^{2} \sin ^{2} \theta_{03}
$$

and

$$
\widehat{V}=\operatorname{diag}\left\{0, V_{e}+V_{n}, V_{n}\right\} .
$$

Eq. (2.27) is obtained from (2.12) by approximating $\cos ^{2} \theta_{13}=1$. Since $\sin ^{2} 2 \theta_{13} \approx 0.09$ and $\sin ^{2} \theta_{13} \approx 0.022$ this is a valid approximation. $\Delta \widehat{m}_{01}^{2}$ is basically the parameter governing the solar $\nu_{e}$ disappearance to $\nu_{s}$ discussed in [2]. Since we will set $\theta_{03}=0$, we will not differentiate between $\Delta m_{01}^{2}$ and $\Delta \widehat{m}_{01}^{2}$, and will always use $\Delta m_{01}^{2}$ in the following of the present article. 

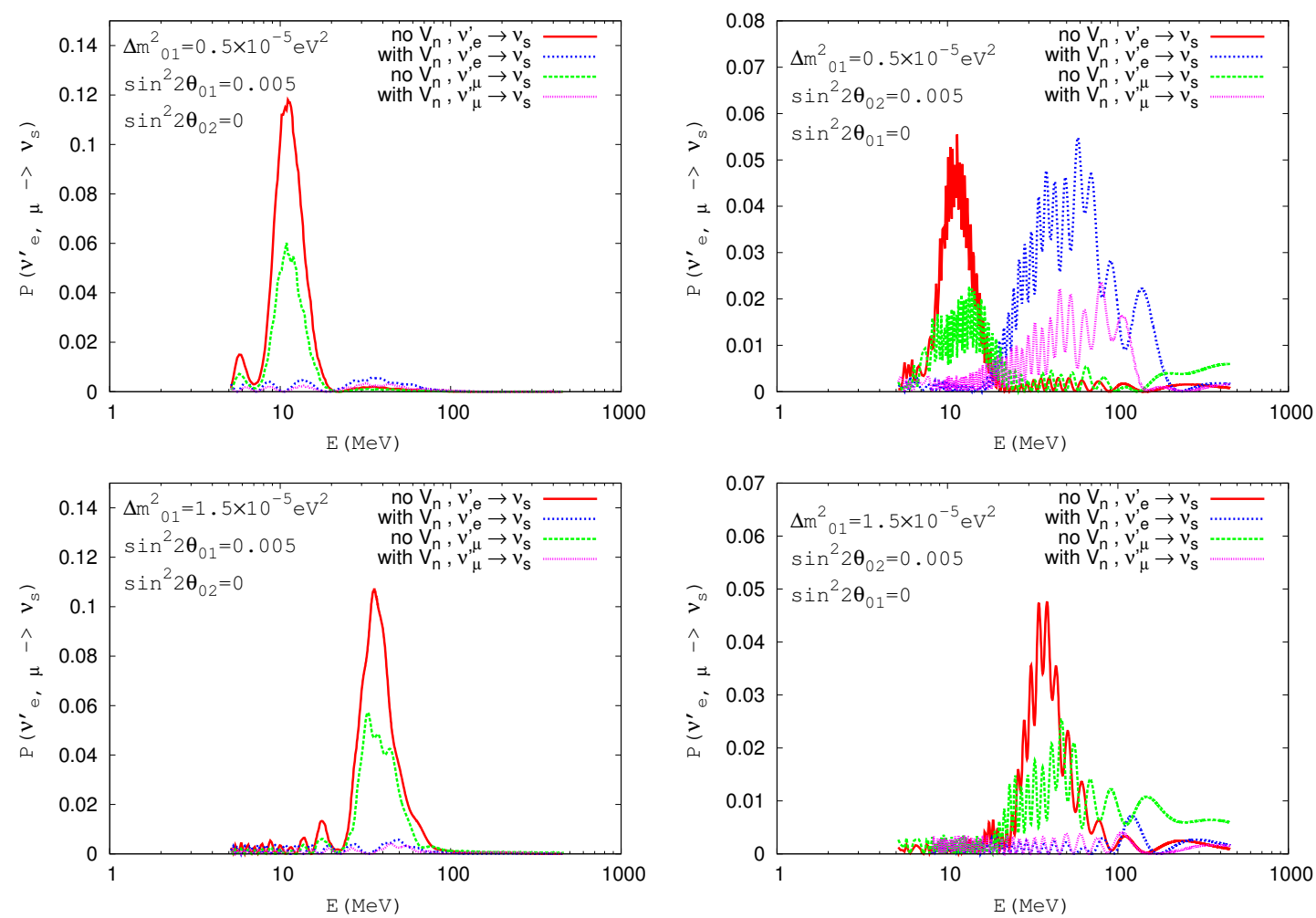

Figure 2. $\nu_{e}^{\prime}-\nu_{s}$ and $\nu_{\mu}^{\prime}-\nu_{s}$ conversion probability versus energy in three neutrino framework both for $V_{n}$ included and for $V_{n}$ switched off. $L=12000 \mathrm{~km}$.

Figure 1 is given with the effect of $V_{n}$ included and has been shown to a particular energy that $\nu_{s}-\nu_{e}^{\prime}$ conversion is close to maximal. In figure 2 we give plots of the conversion probability of $\nu_{e, \mu}^{\prime} \rightarrow \nu_{s}$ versus energy both for $V_{n}$ included and for $V_{n}$ switched off. For the case with the effect of $V_{n}$ included the neutron number density is calculated using (2.17). We can see that for the case with the effect of $V_{n}$ included the probability of $\nu_{e}^{\prime} \rightarrow \nu_{s}$ conversion is maximally around $5 \%$. This maximal conversion probability happens for $\Delta m_{01}^{2} \approx 0.5 \times 10^{-5} \mathrm{eV}^{2}, \sin ^{2} 2 \theta_{02}=0.005$ and $E \approx 60 \mathrm{MeV}$, as can be seen in the upperright plots of figure 2 . The parameters for figure 1 are chosen accordingly in this range of parameter space. For smaller $\sin ^{2} 2 \theta_{02}$ the amplitude of the conversion probability is smaller. For $\Delta m_{01}^{2}=1.5 \times 10^{-5} \mathrm{eV}^{2}$, the conversion probability is maximally around $10^{-3}$ when effect of $V_{n}$ included and the resonant conversion disappears.

As a comparison, we can see in figure 2 that there are indeed much stronger resonant $\nu_{s}-\left(\nu_{e}^{\prime}, \nu_{\mu}^{\prime}\right)$ conversions when $V_{n}$ is switched off. In particular, there is a resonant conversion at energy around $10 \mathrm{MeV}$ when $\Delta m_{01}^{2}$ is around $0.5 \times 10^{-5} \mathrm{eV}^{2}$ and the effect of $V_{n}$ is switched off. This is consistent with our expectation. Fortunately, this resonance disappears after including effect of $V_{n}$. Although there is still a resonant enhancement of $\nu_{e, \mu}^{\prime} \rightarrow \nu_{s}$ conversion for $\Delta m_{01}^{2}=0.5 \times 10^{-5} \mathrm{eV}^{2}$ and $\sin ^{2} 2 \theta_{02}=0.005$ when effect of $V_{n}$ included, the conversion probability can only reach about $5 \%$ and furthermore it appears at energy around $60 \mathrm{MeV}$ which is well beyond the solar neutrino and supernovae neutrino spectrum. For larger $\Delta m_{01}^{2}$ this enhancement disappears completely, as can be seen in the 
plot for $\Delta m_{01}^{2}=1.5 \times 10^{-5} \mathrm{eV}^{2}$ in figure 2 . More details about the variation with respect to $\Delta m_{01}^{2}$ will be presented in the next section.

This is a very interesting observation. If the resonant conversion happens as in the case with $V_{n}$ switched off, active neutrinos of energy around 10 to $60 \mathrm{MeV}$ can oscillate to this super-light sterile. In particular, this could lead to spectrum distortion of high energy solar boron neutrino and the flux of low energy atmospheric active neutrinos should have a dip at this energy range. The disappearance or the suppression of the resonant conversion in the case with $V_{n}$ included shows that the scenario of super-light sterile neutrino can pass through the possible constraints from solar and atmospheric neutrino experiments.

We note that this phenomenon does not happen for the conversion of neutrino in the Sun since the neutron number density is small in the Sun and the effect of $V_{n}$ is indeed switched off. On the other hand, the neutron number density is of the same order of the magnitude of the electron number density in the Earth and the effect of $V_{n}$ can play important role. In the next section we show in detail how the resonance disappears in the Earth matter.

\section{Energy Levels and the flavor conversion of the super-light sterile neu- trino}

In this section we study the effect of $V_{n}$ on the energy levels of neutrinos and on the resonant conversion of the super-light sterile neutrino $\nu_{s}$ with active neutrinos $\nu_{e}^{\prime}$ and $\nu_{\mu}^{\prime}$ in the Earth. We will work in the $3 \nu$ framework as given in eqs. (2.23), (2.24), (2.25), (2.26) and (2.27).

A MSW resonance of flavor conversion happens when two energy levels become close to each other. If a resonant conversion happens, a small mixing in vacuum can effectively lead to large flavor conversion in matter. To examine whether there is a resonant flavor conversion it's crucial to check whether two eigenvalues of the Hamiltonian $\widehat{H}$ can be close to each other.

As will be shown below, it's sufficient to set small parameters $\theta_{01}$ and $\theta_{02}$ to zero when studying the energy levels of $\widehat{H}$. For convenience we will set $\theta_{01}=\theta_{02}=0$ and discuss the energy levels of the following Hamiltonian

$$
\widehat{H}=\left(\begin{array}{ccc}
\frac{\Delta m_{01}^{2}}{2 E} & 0 & 0 \\
0 & \frac{\Delta m_{21}^{2}}{2 E} \sin ^{2} \theta_{12}+V_{e}+V_{n} & \frac{\Delta m_{21}^{2}}{2 E} \sin \theta_{12} \cos \theta_{12} \\
0 & \frac{\Delta m_{21}^{2}}{2 E} \sin \theta_{12} \cos \theta_{12} & \frac{\Delta m_{21}^{2}}{2 E} \cos ^{2} \theta_{12}+V_{n}
\end{array}\right) .
$$

Eq. (3.1) is obtained from (2.24) by setting $\theta_{01}=\theta_{02}=0$ in $\hat{U}$. The convenience of using (3.1) is clear by noting that the three eigenvalues of (3.1) can be easily found as

$$
\begin{aligned}
& E_{0}=\frac{\Delta m_{01}^{2}}{2 E} \\
& E_{1}=\frac{1}{2}\left[\frac{\Delta m_{21}^{2}}{2 E}+V_{e}-\sqrt{\left(\frac{\Delta m_{21}^{2}}{2 E} \cos 2 \theta_{12}-V_{e}\right)^{2}+\left(\frac{\Delta m_{21}^{2}}{2 E} \sin 2 \theta_{12}\right)^{2}}\right]+V_{n}, \\
& E_{1}=\frac{1}{2}\left[\frac{\Delta m_{21}^{2}}{2 E}+V_{e}+\sqrt{\left(\frac{\Delta m_{21}^{2}}{2 E} \cos 2 \theta_{12}-V_{e}\right)^{2}+\left(\frac{\Delta m_{21}^{2}}{2 E} \sin 2 \theta_{12}\right)^{2}}\right]+V_{n} .
\end{aligned}
$$



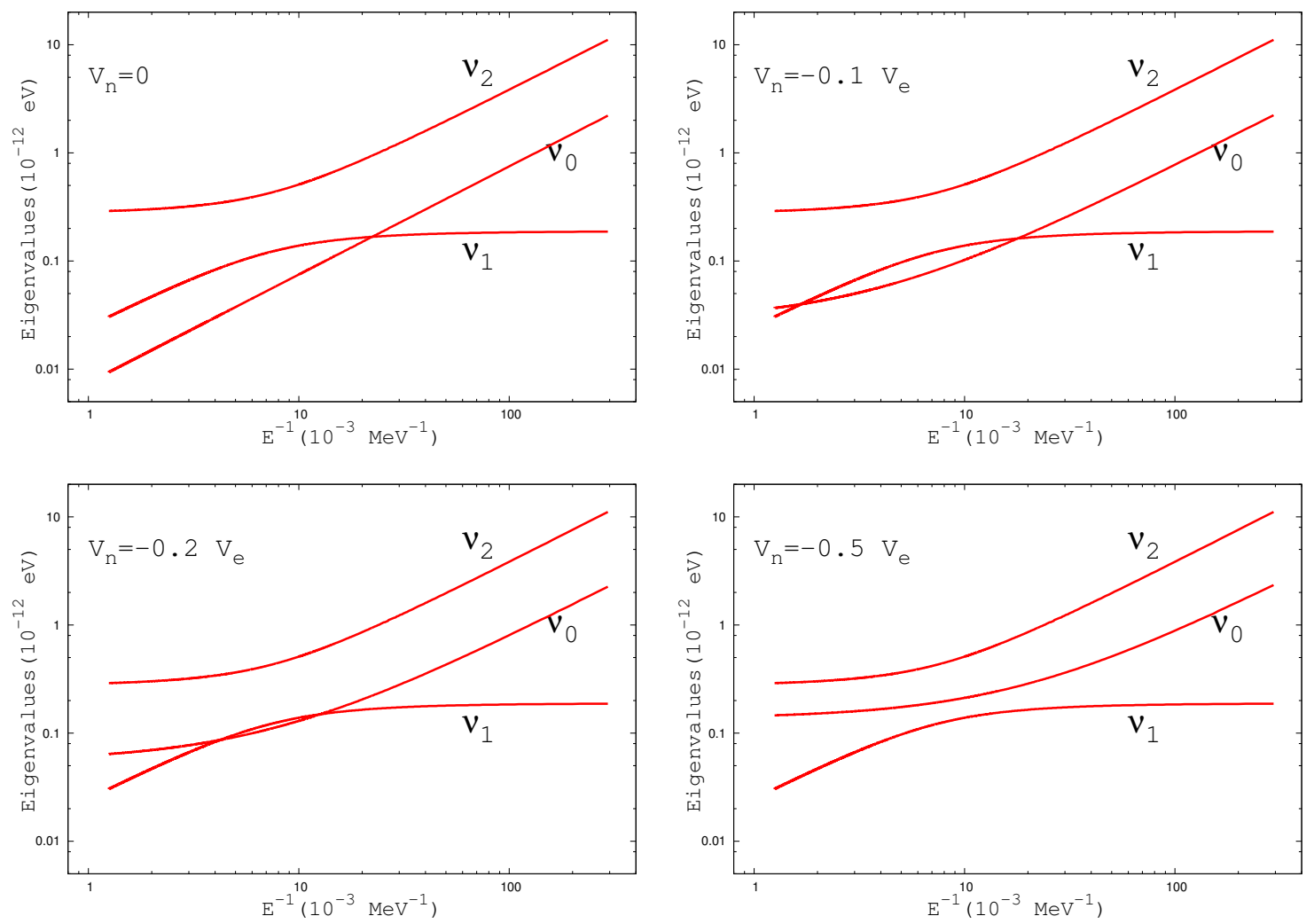

Figure 3. Energy levels $\nu_{0}, \nu_{1}$ and $\nu_{2}$ versus $E^{-1}$ in three neutrino framework. Three lines for $\nu_{0}$, $\nu_{1}$ and $\nu_{2}$ are $E_{0}-V_{n}, E_{1}-V_{n}$ and $E_{2}-V_{n}$ separately. $\theta_{01}=\theta_{02}=0, \Delta m_{01}^{2}=1.5 \times 10^{-5} \mathrm{eV}^{2}$, $\Delta m_{21}^{2}=7.5 \times 10^{-5} \mathrm{eV}^{2}, \sin ^{2} 2 \theta_{12}=0.857, V_{e}=2.74 \times 10^{-13} \mathrm{eV}$.

$E_{0}, E_{1}, E_{2}$ are eigenvalues of (3.1) corresponding to neutrinos in mass base $\nu_{0}, \nu_{1}$ and $\nu_{2}$ separately. To illustrate qualitatively the effect of the Earth matter on the oscillation of super-light sterile neutrino $\nu_{s}$ with active neutrinos $\nu_{e}^{\prime}$ and $\nu_{\mu}^{\prime}$, it's convenient to compute $E_{1}$ and $E_{2}$ using a trajectory dependent averaged potential [20]

$$
\bar{V}_{e}=\frac{1}{L} \int_{0}^{L} d x V_{e}(x)
$$

where $L$ is the length of the neutrino trajectory in the Earth. For baseline longer than $6000 \mathrm{~km}, \bar{V}_{e}$ varies from $1.6 \times 10^{-13} \mathrm{eV}$ to about $2.7 \times 10^{-13} \mathrm{eV}$. As will be detailed in the next section a formulation using the average potential (3.5) gives a pretty good description of the oscillation of super-light sterile neutrino with active neutrinos.

In figure 3 we give plots for $E_{i}-V_{n}$ and compare different cases with various $V_{n}$. For $V_{n}=0$ and $\Delta m_{01}^{2}=1.5 \times 10^{-5} \mathrm{eV}^{2}$, two eigenvalues $E_{0}$ and $E_{1}$ can get close to each other at energy around $40 \mathrm{MeV}$ and we can see that two lines actually cross at a point in this energy range. There should be resonant conversion of neutrinos in this energy range. We can see in lower plots in figure 2 that for $\Delta m_{01}^{2}=1.5 \times 10^{-5} \mathrm{eV}^{2}$ there are indeed resonant flavor conversions at $E \approx 40 \mathrm{MeV}$ when effect of $V_{n}$ is switched off. 

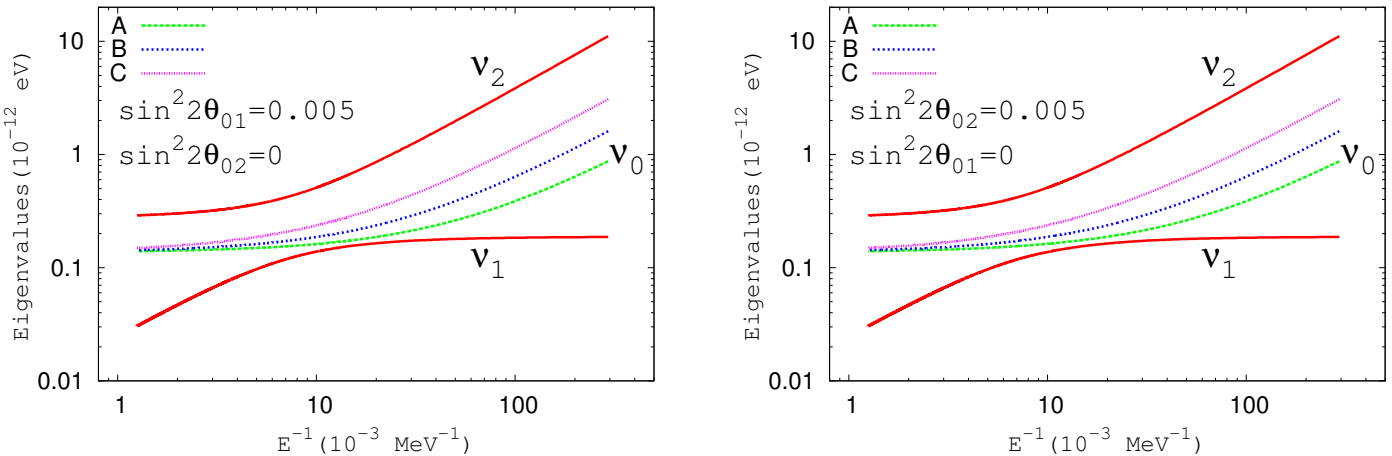

Figure 4. $E_{0}-V_{n}, E_{1}-V_{n}$ and $E_{2}-V_{n}$ for $\nu_{0}, \nu_{1}$ and $\nu_{2}$ versus $E^{-1}$ in three neutrino framework. $V_{n}=-0.5 V_{e}$ and $V_{e}=2.74 \times 10^{-13} \mathrm{eV}$. Lines A, B and $\mathrm{C}$ correspond to cases with $\Delta m_{01}^{2}=$ $0.5 \times 10^{-5} \mathrm{eV}^{2}, \Delta m_{01}^{2}=1.0 \times 10^{-5} \mathrm{eV}^{2}$ and $\Delta m_{01}^{2}=2.0 \times 10^{-5} \mathrm{eV}^{2}$ separately.

Increasing the magnitude of $V_{n}$ first gives rise to a second region for resonant conversion, as can be seen in the plot with $V_{n}=-0.1 V_{e}$. But for $V_{n}=-0.5 V_{e}$ there is no chance for the two lines of $E_{0}$ and $E_{1}$ become as close to each other as that for $V_{n}=0$, and the resonance should disappear or be significantly suppressed compared to the case with $V_{n}=0$. We note that this is exactly the situation we have in the Earth matter. As can be seen in eq. (2.17), the neutron number density is roughly equal to the electron number density in the Earth and hence $V_{n} \approx-0.5 V_{e}$. This explains why the flavor conversion for $\Delta m_{01}^{2}=1.5 \times 10^{-5} \mathrm{eV}^{2}$, as shown in lower plots in figure 2 , is strongly suppressed when effect of $V_{n}$ is included.

For a non-zero but small $\theta_{01}$ or $\theta_{02}$ one can give similar plots for $E_{0}, E_{1}$ and $E_{2}$ and there are no visible differences compared to the plots in figure 3. So the above discussion presented for $\theta_{01}=\theta_{02}=0$ can be applied to the case with non-zero but small $\theta_{01}$ or $\theta_{02}$, and the reason of the absence of resonance for a relatively large value of $\Delta m_{01}^{2}$ is clear according to discussion presented above. In figure 4 we give plots of $E_{i}-V_{n}$ for $\theta_{01,02} \neq 0$. $E_{i}$ are obtained from diagonalizing (2.24) numerically. $V_{n} / V_{e}$ is fixed in figure 4 while $\Delta m_{01}^{2}$ varies. In Earth matter $V_{e} / V_{n}$ can vary from -0.5012 to -0.573 . As can be seen in figure 3, the larger the $\left|V_{e} / V_{n}\right|$ is, the farther away from each other the two energy levels. So we choose $V_{e}=-0.5 V_{n}$ in figure 4 . We can see that for $\Delta m_{01}^{2}=(0.5-2) \times 10^{-5} \mathrm{eV}^{2}$, which is the parameter space suggested in ref. [2], the line of $E_{0}$ is always in-between the two lines of $E_{1}$ and $E_{2}$. The only possible case for a resonant conversion to happen is when $\Delta m_{01}^{2} \approx 0.5 \times 10^{-5} \mathrm{eV}^{2}$. In this case $E_{0}$ and $E_{1}$ come close to each other at $E \approx 60 \mathrm{MeV}$. However, even in this case the $\nu_{e, \mu}^{\prime} \rightarrow \nu_{s}$ conversion probability is maximally around $5 \%$ when $\sin ^{2} 2 \theta_{02}=0.005$, as has been shown in figure 2 .

In figure 5 we give plots of $E_{i}-V_{n}$ similar to figure 4 but with different $V_{e}$. We can see that the situation is very similar to that in figure 4 and energy levels $E_{0}$ and $E_{1}$ cannot become close to each other unless $\Delta m_{01}^{2}$ is close to $0.5 \times 10^{-5} \mathrm{eV}^{2}$. From the above discussion we can conclude that due to the presence of $V_{n}$ in Earth matter the naively expected resonant conversion should disappear or strongly suppressed.

In figure 6 we show the variation of the probability of $\nu_{e, \mu}^{\prime} \rightarrow \nu_{s}$ conversion with respect to $\Delta m_{01}^{2}$. We can see that as $\Delta m_{01}^{2}$ increases from the value $0.5 \times 10^{-5} \mathrm{eV}^{2}$, the amplitude 

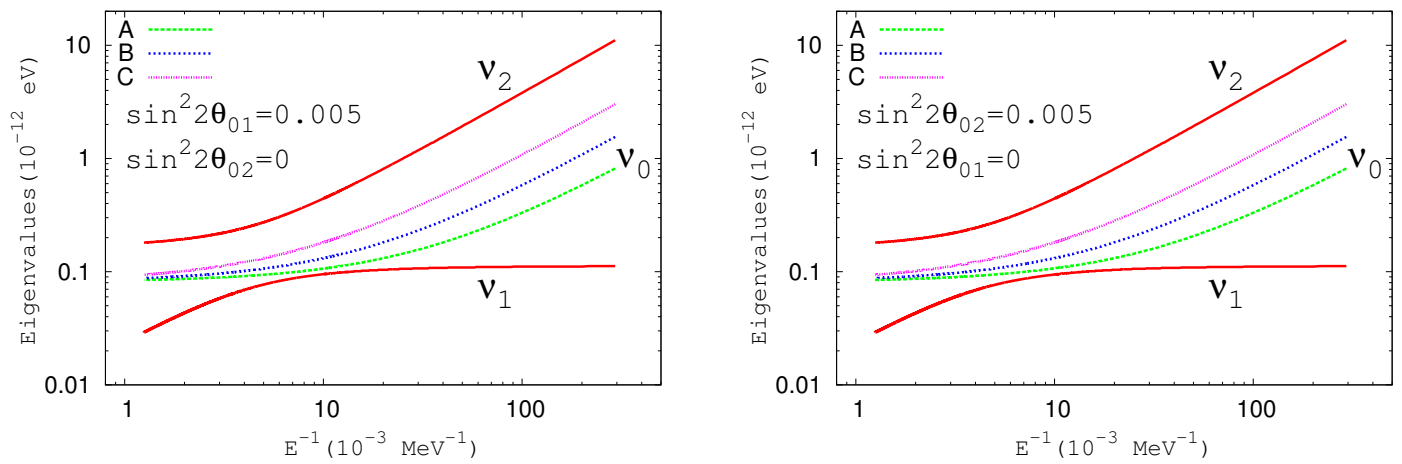

Figure 5. $E_{0}-V_{n}, E_{1}-V_{n}$ and $E_{2}-V_{n}$ for $\nu_{0}, \nu_{1}$ and $\nu_{2}$ versus $E^{-1}$ in three neutrino framework. $V_{n}=-0.5 V_{e}$ and $V_{e}=1.63 \times 10^{-13} \mathrm{eV}$. Lines A, B and $\mathrm{C}$ correspond to cases with $\Delta m_{01}^{2}=$ $0.5 \times 10^{-5} \mathrm{eV}^{2}, \Delta m_{01}^{2}=1.0 \times 10^{-5} \mathrm{eV}^{2}$ and $\Delta m_{01}^{2}=2.0 \times 10^{-5} \mathrm{eV}^{2}$ separately.
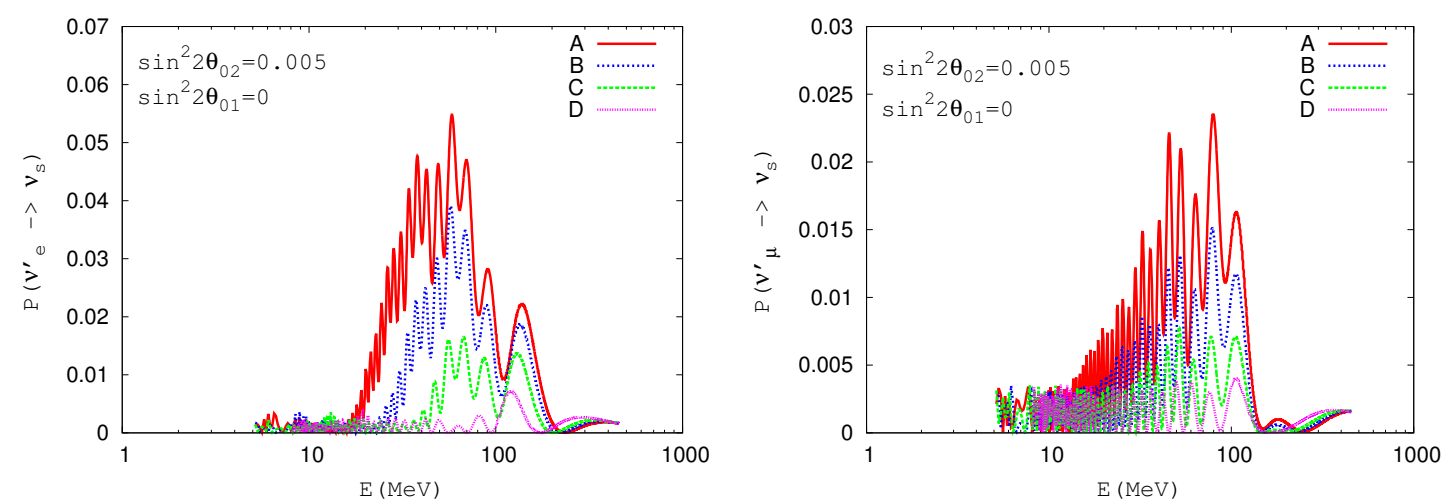

Figure 6. $\nu_{e}^{\prime} \rightarrow \nu_{s}$ and $\nu_{\mu}^{\prime} \rightarrow \nu_{s}$ conversion probability versus energy. Lines A, B, C and D correspond to $\Delta m_{01}^{2}=0.5 \times 10^{-5} \mathrm{eV}^{2}, \Delta m_{01}^{2}=0.7 \times 10^{-5} \mathrm{eV}^{2}, \Delta m_{01}^{2}=1.0 \times 10^{-5} \mathrm{eV}^{2}, \Delta m_{01}^{2}=$ $1.5 \times 10^{-5} \mathrm{eV}^{2}$ separately. $L=12000 \mathrm{~km}$.

of the conversion probability decreases and for $\Delta m_{01}^{2}>1.0 \times 10^{-5} \mathrm{eV}^{2}$ the probability is maximally around $1 \%$. We see that the resonant conversion disappears for a relatively large value of $\Delta m_{01}^{2}$.

The above discussion have been presented for disappearance of active neutrinos to the super-light sterile neutrino. For $\nu_{s} \rightarrow \nu_{e, \mu}^{\prime}$, the appearance of active neutrinos from the sterile neutrino, we can study similarly. In the case under study we have no $\mathrm{CP}$ violating phase in mixing matrix $\hat{U}$ and we should have $P\left(\nu_{s} \rightarrow \nu_{e, \mu}^{\prime}\right)=P\left(\nu_{e, \mu}^{\prime} \rightarrow \nu_{s}\right)$. So the previous discussion presented for $P\left(\nu_{e, \mu}^{\prime} \rightarrow \nu_{s}\right)$ can be directly translated to $P\left(\nu_{s} \rightarrow \nu_{e, \mu}^{\prime}\right)$.

For solar neutrinos one can similarly show that if $n_{n} / n_{e} \gtrsim 0.9$ in the Sun the situation would be similar to that in the Earth. For real matter density in the Sun which has a negligible neutron number density, the level crossing and the MSW resonance for the super-light sterile neutrino indeed exist.

The above discussions of oscillation have been presented for $\nu_{l}=\left(\nu_{s}, \nu_{e}^{\prime}, \nu_{\mu}^{\prime}\right)$. Solar neutrinos arrive at the Earth as mass eigenstates, say as $\nu_{0}, \nu_{1}$ or $\nu_{2}$. The probability of 
the conversion of $\nu_{i}$, a neutrino in mass eigenstate, to $\nu_{l}$, can be calculated as

$$
P\left(\nu_{i} \rightarrow \nu_{l}\right)=\left|\sum_{l^{\prime}} \hat{U}_{l^{\prime} i} M\left(\nu_{l^{\prime}} \rightarrow \nu_{l}\right)\right|^{2} .
$$

where $\nu_{l^{\prime}}$ runs over $\nu_{s}, \nu_{e}^{\prime}$ and $\nu_{\mu}^{\prime}$ and $M\left(\nu_{l^{\prime}} \rightarrow \nu_{l}\right)$ is the evolution matrix calculated in the framework of eqs. (2.23) and (2.24). For example, for $\nu_{0} \rightarrow \nu_{e}^{\prime}$ conversion we have

$$
P\left(\nu_{0} \rightarrow \nu_{e}^{\prime}\right)=\left|\hat{U}_{s 0} M\left(\nu_{s} \rightarrow \nu_{e}^{\prime}\right)+\hat{U}_{e 0} M\left(\nu_{e}^{\prime} \rightarrow \nu_{e}^{\prime}\right)+\hat{U}_{\mu 0} M\left(\nu_{\mu}^{\prime} \rightarrow \nu_{e}^{\prime}\right)\right|^{2} .
$$

According to previous discussions, $P\left(\nu_{s} \rightarrow \nu_{e}^{\prime}\right)=\left|M\left(\nu_{s} \rightarrow \nu_{e}^{\prime}\right)\right|^{2}$ is maximally around $10^{-3}$ for solar neutrinos which have energy $\lesssim 18 \mathrm{MeV}$. Hence, we can find that three terms in eq. (3.7) which contribute to the amplitude are all suppressed for the case $\left(\left|\hat{U}_{e 0}\right|^{2},\left|\hat{U}_{\mu 0}\right|^{2}\right) \lesssim 10^{-3}$ ( or $\left|\theta_{01}\right|^{2}$ and $\left|\theta_{02}\right|^{2}$ less than about $10^{-3}$ ) and eq. (3.7) is maximally at order $10^{-3}$ for solar neutrinos. Numerical calculation agrees with this estimate. Similarly, one can find that the correction to $P\left(\nu_{1,2} \rightarrow \nu_{e}^{\prime}\right)$ due to mixing with $\nu_{s}$ is negligible. For oscillation to $\nu_{\mu}^{\prime}$ the conclusion is similar. The fluxes of the solar electron neutrino or muon(tau) neutrino can be calculated using the fluxes of the $\nu_{i}$ neutrino and the probability $P\left(\nu_{i} \rightarrow \nu_{e}^{\prime}\right)$ or $P\left(\nu_{i} \rightarrow \nu_{\mu}^{\prime}\right)$. It's straightforward to show that the regeneration effect due to Earth matter is dominated by that in the $1-2$ oscillation and the correction due to the mixing with the super-light sterile neutrino is negligible.

\section{Formulation of oscillation of super-light sterile neutrino in the Earth}

In this section we present a perturbation theory describing the oscillation of super-light sterile neutrino with active neutrinos in the Earth matter. This formulation uses the baseline averaged potential (3.5) and its basic strategy is the same as that presented for oscillation among active neutrinos in [20]. We will show that the leading term in the theory, which is analytic, gives a qualitatively good description of the flavor conversion of the super-light sterile neutrino $\nu_{s}$ with the active neutrinos. Including the first order correction this perturbation theory is precise to describe the flavor conversion between $\nu_{s}$ and $\nu_{e, \mu}^{\prime}$. This justifies the use of the average potential (3.5) in the discussion of the last section. The theory is detailed below.

Similar to the formulation in [20], we can introduce an average potential for a trajectory of neutrino with a baseline length $L$ in the Earth

$$
\bar{V}=\operatorname{diag}\left\{0, \bar{V}_{e}+\bar{V}_{n}, \bar{V}_{n}\right\}=\frac{1}{L} \int_{0}^{L} d x \widehat{V}(x),
$$

where $\bar{V}_{e}$ has been introduced in (3.5) and $\bar{V}_{n}$ is similarly defined. The Hamiltonian (2.24) can be rewritten using (4.1) as

$$
\widehat{H}(x)=\bar{H}+\delta \widehat{H}(x),
$$

where

$$
\bar{H}=\hat{U} \widehat{H}_{0} \hat{U}^{\dagger}+\bar{V},
$$



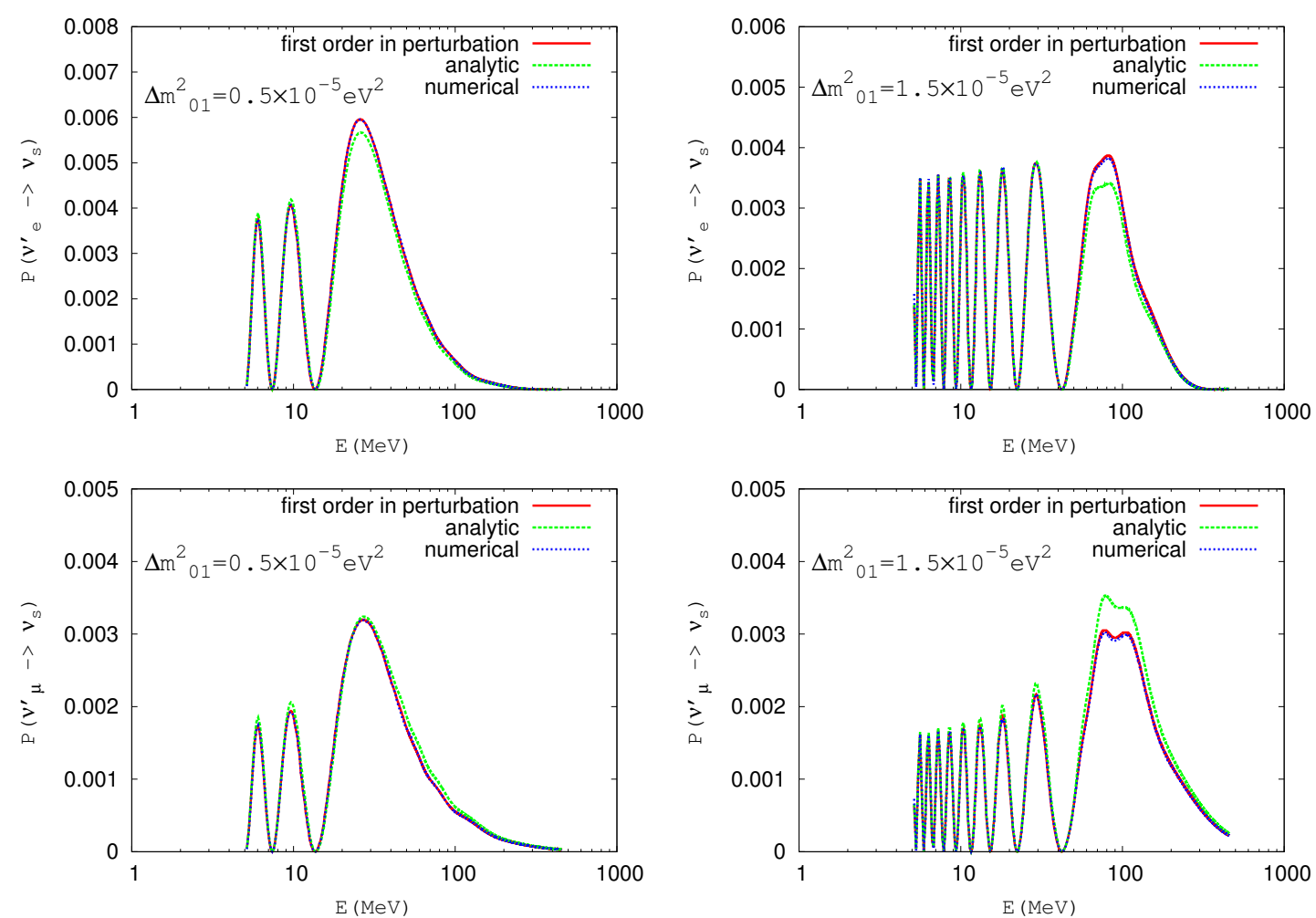

Figure 7. $P\left(\nu_{e}^{\prime}, \nu_{\mu}^{\prime} \rightarrow \nu_{s}\right)$ versus energy for $L=8000 \mathrm{~km} \cdot \sin ^{2} 2 \theta_{01}=0.005, \theta_{02}=0$.

and $\delta \widehat{H}=\widehat{H}-\bar{H}$ which equals to $\delta \widehat{V}$

$$
\delta \widehat{H}=\delta \widehat{V}=\widehat{V}-\bar{V}
$$

Introducing a mixing matrix $U_{m}$ in matter which diagonalizes $\bar{H}$ :

$$
\bar{H} U_{m}=U_{m} \frac{1}{2 E} \Delta, \quad \Delta=\operatorname{diag}\left\{\Delta^{1}, \Delta^{2}, \Delta^{3}\right\},
$$

where $\frac{1}{2 E} \Delta^{i}(i=1,2,3)$ are three eigenvalues of $\bar{H}$, we are ready to solve the evolution problem in (2.23) perturbatively in an expansion in $\delta \widehat{V}$. We first solve the evolution governed by $\bar{H}$ and obtain the contribution of $\delta V$ using perturbation in $\delta V$. Keeping result of first order in $\delta V$ we obtain

$$
\begin{aligned}
& \widehat{\nu}^{\prime}(L)=M(L) \widehat{\nu}^{\prime}(0), \\
& M(L)=U_{m} e^{-i \frac{\Delta}{2 E} L}(1-i C) U_{m}^{\dagger},
\end{aligned}
$$

where $C$ is a $3 \times 3$ matrix accounting for the non-adiabatic correction

$$
C=\int_{0}^{L} d x e^{i \frac{\Delta}{2 E} x} U_{m}^{\dagger} \delta V(x) U_{m} e^{-i \frac{\Delta(x)}{2 E} x}
$$



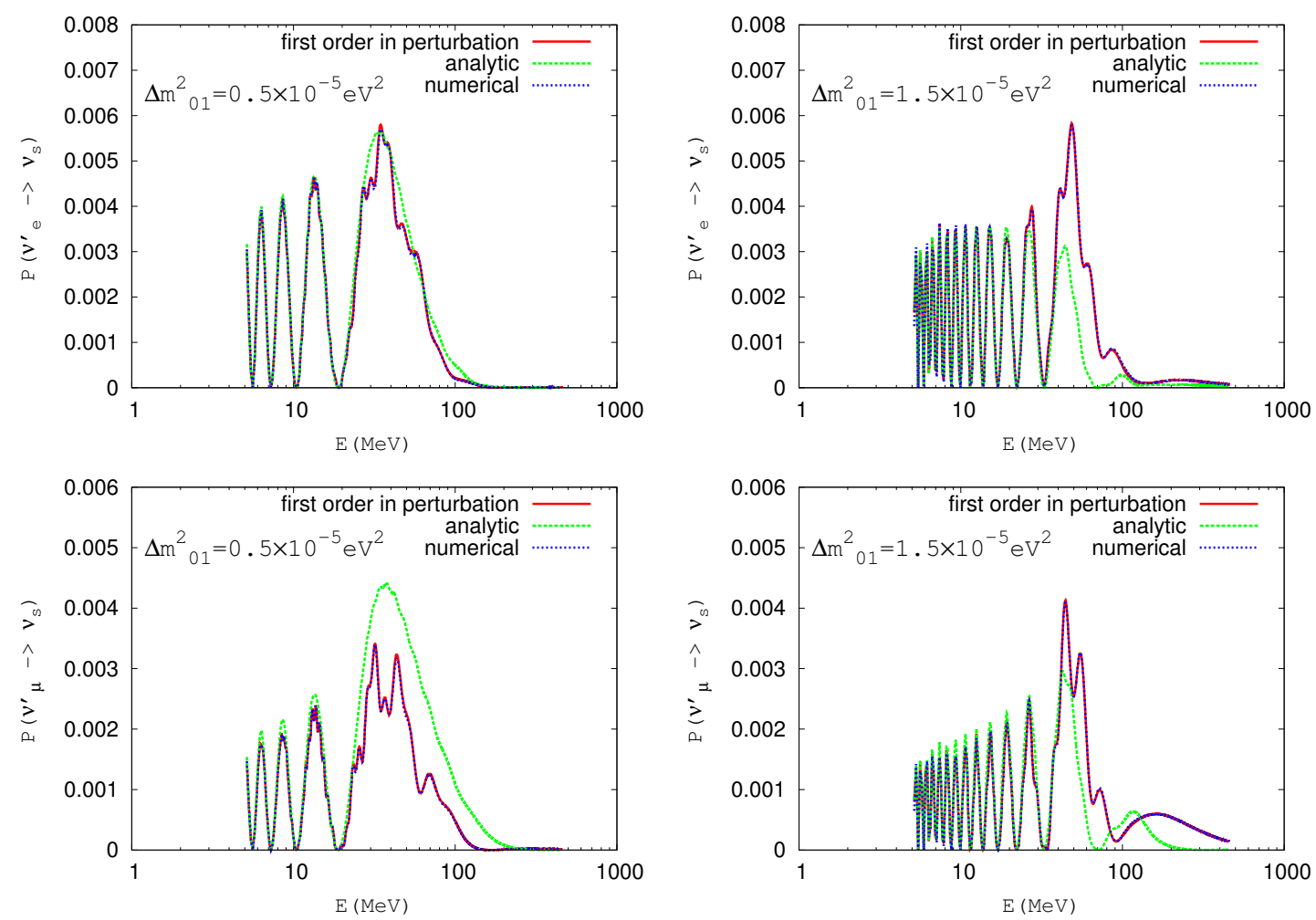

Figure 8. $P\left(\nu_{e}^{\prime}, \nu_{\mu}^{\prime} \rightarrow \nu_{s}\right)$ versus energy for $L=12000 \mathrm{~km} . \sin ^{2} 2 \theta_{01}=0.005, \theta_{02}=0$.

It is clear that $C^{\dagger}=C$ holds. One can see that

$$
\begin{aligned}
C_{j j} & =\int_{0}^{L} d x\left(U_{m}^{\dagger} \delta V(x) U_{m}\right)_{j j}=0, \\
C_{j k} & =\int_{0}^{L} d x e^{i \frac{\Delta^{j}-\Delta^{k}}{2 E} x}\left(U_{m}^{\dagger} \delta V(x) U_{m}\right)_{j k}, \quad j \neq k .
\end{aligned}
$$

eq. (4.9) is guaranteed by eq. (4.1). $\left|C_{j k}\right| \ll 1(j \neq k)$ should be satisfied if this is a good perturbation theory. One of the virtues of this perturbation theory is that eq. (4.9) guarantees that the oscillation phase is correctly reproduced.

In figure 7 we give plots for $\nu_{s}-\nu_{e}^{\prime}$ conversion versus energy for $L=8000 \mathrm{~km}$. We give zero-th order results calculated using analytic formula, i.e. using (4.6) with $C$ switched off. We also present the result including the first order correction (4.8). The line for numerical result is calculated using the PREM Earth density profile [21]. We can see that the zeroth order result using trajectory averaged potential reproduces the oscillation phase very well, but not the magnitude. Including the first order correction calculated using (4.8), this formalism reproduces very well the oscillation phase and magnitude of the flavor conversion.

For $L>10690 \mathrm{~km}$ neutrinos cross the core of the Earth. Large density jump between the core and the mantle makes the above simple version of the perturbation theory not as precise as shown in figure 7 . We can improve the approximation by dividing the whole trajectory into three parts with parts 1 and 3 in the mantle and part 2 in the core of the 

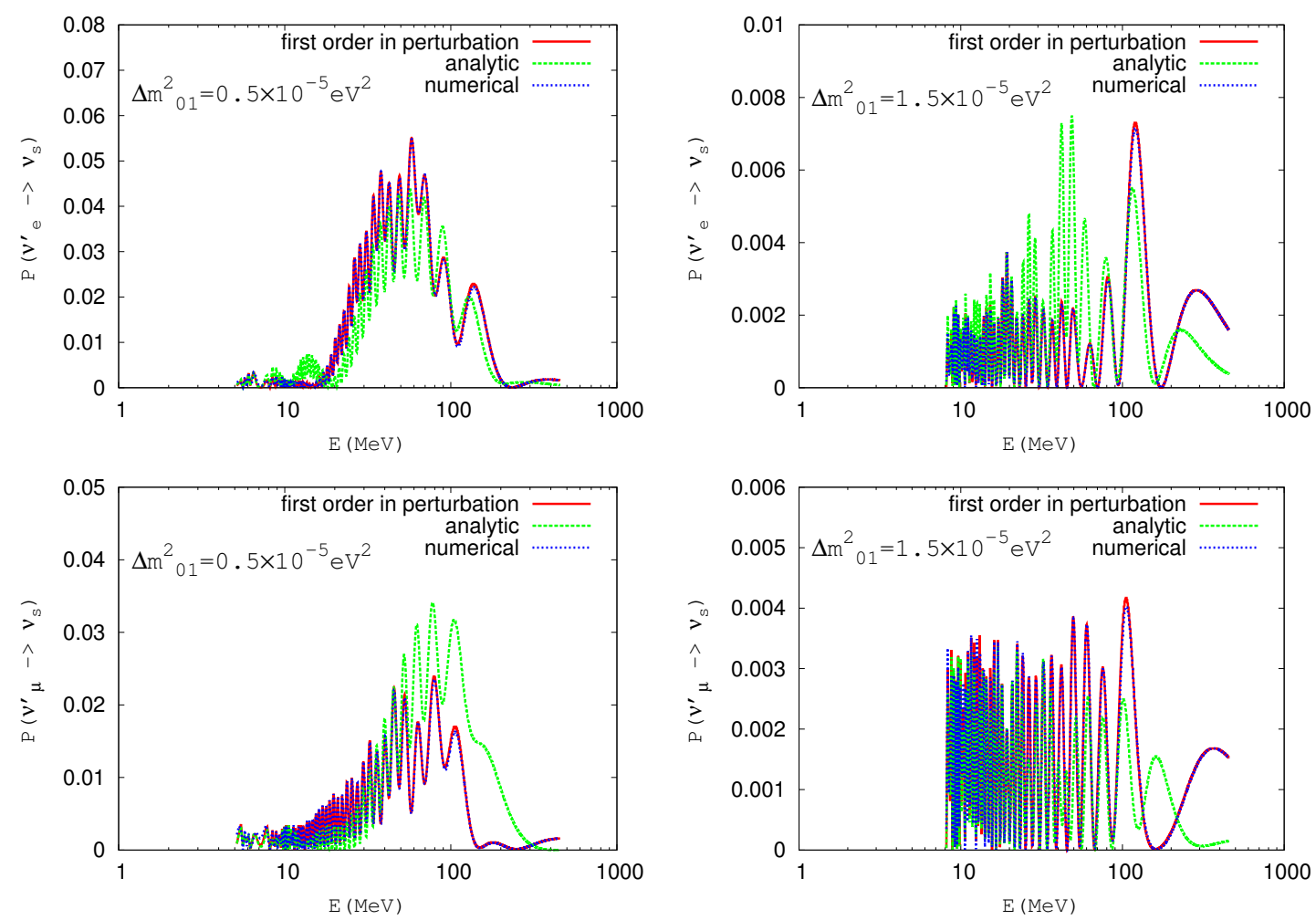

Figure 9. $P\left(\nu_{e}^{\prime}, \nu_{\mu}^{\prime} \rightarrow \nu_{s}\right)$ versus energy for $L=12000 \mathrm{~km} . \sin ^{2} 2 \theta_{02}=0.005, \theta_{01}=0$.

Earth. The evolution matrix can be written as

$$
M=M_{3} M_{2} M_{1}
$$

where $M_{2}$ is the evolution matrix in the core and $M_{1,3}$ are evolution matrices in the mantle. $M_{i}(i=1,2,3)$ are calculated similar to (4.6) but with average potentials evaluated in the corresponding part of the trajectory in the mantle and in the core separately [20]. In figure 8 we give plots for an example of this case. We can see that the result calculated using the improved perturbation theory (4.11) agrees well with the numerical result. The oscillation phase and the magnitude of the flavor conversion are very well reproduced in the perturbation theory. We also give the analytic result calculated using the potential averaged over the whole trajectory (4.1). We can see that this analytic result correctly reproduces the oscillation phase of the $\nu_{s}-\nu_{e}^{\prime}$ conversion.

In figure 9 and 10 we give plots of $\nu_{e, \mu}^{\prime} \rightarrow \nu_{s}$ oscillation similar to figure 7 and 8, but for non-zero $\theta_{02}$. Similarly, we can see that the analytic result gives a good account of the phase of the oscillation of the super-light sterile neutrino with active neutrinos. The peterubation theory gives not only reproduces the oscillation phase but also the magnitude of the flavor conversion very well.

We note that the above discussion does not mean that the perturbation theory presented in this article give a precise description for $1-2$ oscillation. The theory presented in this article indeed give a qualitatively good description for $1-2$ oscillation for energy $\gtrsim 20 \mathrm{MeV}$, but not precisely. Actually for an energy around $10 \mathrm{MeV}$ the structure of the 

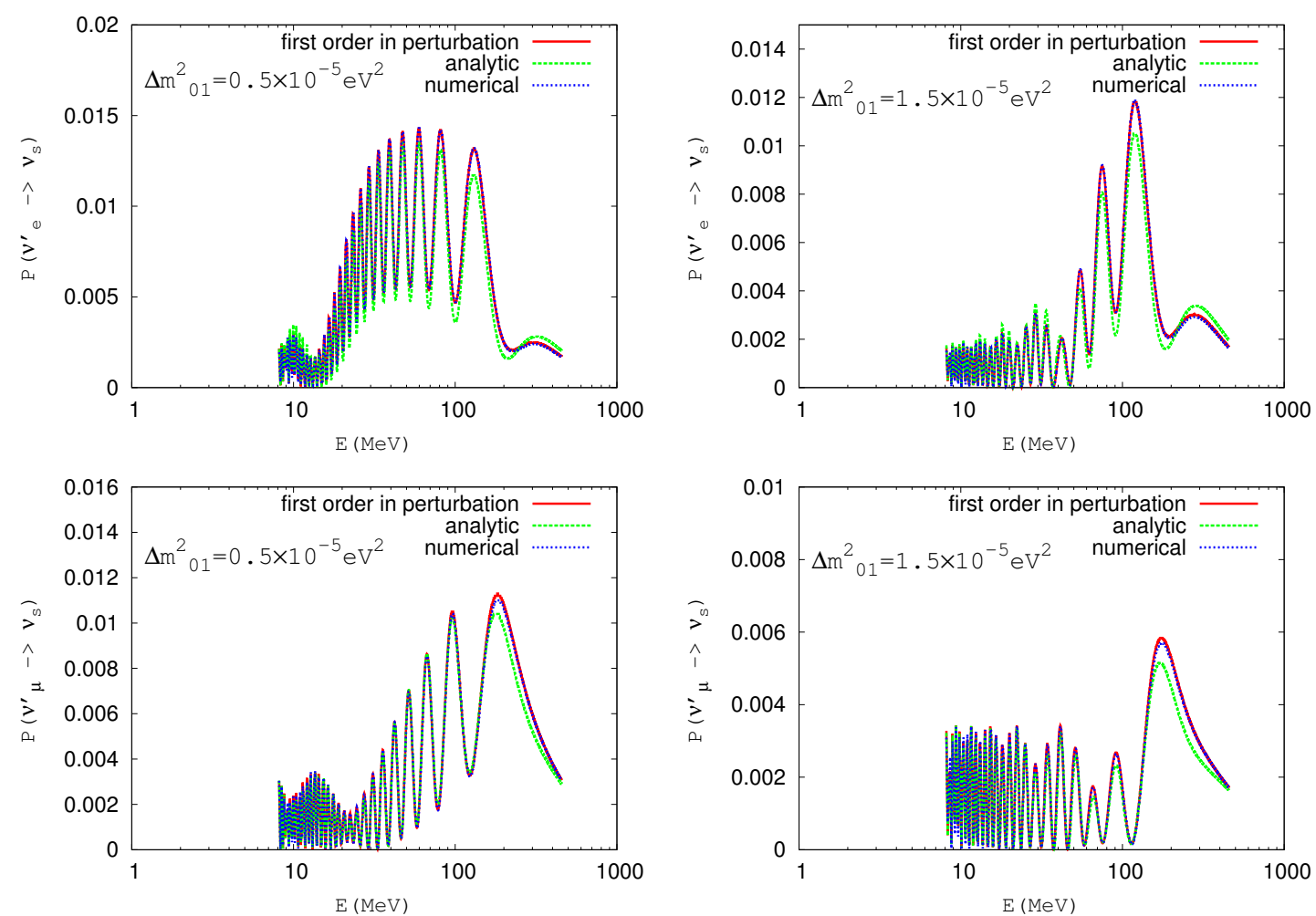

Figure 10. $P\left(\nu_{e}^{\prime}, \nu_{\mu}^{\prime} \rightarrow \nu_{s}\right)$ versus energy for $L=8000 \mathrm{~km} . \sin ^{2} 2 \theta_{02}=0.005, \theta_{01}=0$.

Earth matter shows up in $1-2$ oscillation [22] and the perturbation theory using average potential is not a precise description. This perturbation theory gives a precise description of $1-2$ oscillation for energy $\gtrsim 500 \mathrm{MeV}[20]$.

We can see from the above discussion that the zero-th order result calculated using the trajectory averaged potential, eq. (4.1), always give a correct account of the oscillation phase of the $\nu_{s}-\nu_{e, \mu}^{\prime}$ conversion. Although the zero-th order result does not give a precise description of the magnitude of conversion, it encodes the major properties of the flavor conversion in the Earth. This justifies the use of the average potential in the discussion of the energy levels and the disappearance of the resonance in the last section.

\section{Conclusion}

In summary we have made a detailed study of the flavor conversion of the super-light sterile neutrino with active neutrinos in Earth matter. A super-light sterile neutrino, with a mass squared difference $\Delta m_{01}^{2} \approx(0.5-2) \times 10^{-5} \mathrm{eV}^{2}$ and a small mixing angle $\theta_{01}$ or $\theta_{02}$, can oscillate with electron neutrinos in the Sun through a MSW resonance and can help to explain the absence of the upturn of the solar boron neutrino spectrum at energy $\lesssim 4 \mathrm{MeV}$. One would naively expect that a similar resonant conversion should also happen when neutrinos pass through the Earth. In this article we have shown that for $\Delta m_{01}^{2} \gtrsim 1 \times 10^{-5} \mathrm{eV}^{2}$ this naively expected resonant conversion disappears completely and for smaller value of $\Delta m_{01}^{2}$ there is still an enhancement of the flavor conversion but the conversion probability is at most a few percent. 
We have shown that the absence or the suppression of the resonant conversion is because of the presence of the potential $V_{n}$ which arises from neutral current interaction of active neutrinos with neutrons in matter. The neutron number density in the Sun is negligible comparing to the electron density and the effect of $V_{n}$ is basically switched off. On the other hand, the neutron number density in the Earth roughly equals to the electron number density and the effect of $V_{n}$ can play important role. When including the effect of $V_{n}$ in Earth matter, we find that for $\Delta m_{01}^{2}=(0.5-2) \times 10^{-5} \mathrm{eV}^{2}$ the energy of the super-light sterile neutrino is always in-between the energies of $\nu_{1}$ and $\nu_{2}$ neutrinos and there is no crossing of energy levels among these neutrinos. For $\Delta m_{01}^{2}$ around $0.5 \times$ $10^{-5} \mathrm{eV}^{2}$ the energies of the super-light sterile neutrino and the active neutrino $\nu_{1}$ have a chance to be close to each other when the energy is around $60 \mathrm{MeV}$ and hence create a resonant conversion. However this resonant enhancement of the flavor conversion is significantly suppressed comparing to the case when $V_{n}$ is switched off and the flavor conversion probability is at most a few percent. Furthermore, the position of the resonance is shifted to an energy around $60 \mathrm{MeV}$ which makes this scenario easily be able to pass through possible constraints coming from measurement of Earth matter effect in solar neutrino experiments.

The observation in the present article is interesting. A MSW resonance is needed in explaining the absence of the upturn of the solar boron neutrino at energy $\lesssim 4 \mathrm{MeV}[2]$. However, a resonant conversion of this super-light sterile neutrino with active neutrino is dangerous when confronting this scenario with experiments which can test the Earth matter effect, e.g. the solar and atmospheric neutrino experiments. Interestingly, we find that different situations of neutron number densities in the Sun and in the Earth make the resonant conversion between the super-light sterile neutrino and active neutrino turned on and effectively turned off, respectively.

For completeness of our discussion we have also shown a perturbation theory which makes use of an baseline averaged potential in developing the theory. This theory can very well describe the flavor conversion of the super-light sterile neutrino with active neutrinos in Earth matter. This justifies the use of the baseline averaged potential in the discussion of the level crossing of neutrinos. We have also discussed the reduction of the flavor conversion in the $4 \nu$ framework to a $3 \nu$ framework.

Apparently the scenario of super-light sterile neutrino is difficult to test in groundbased experiment of neutrino oscillation. We have shown that the conversion of $\nu_{e, \mu}^{\prime} \rightarrow \nu_{s}$ is maximally a few percent and the maximal conversion happens for energy around $60 \mathrm{MeV}$. This energy range is well beyond that of the solar and supernovae neutrino spectrum. The only possibility is to test it in very low energy atmospheric neutrino data. However, this is also pretty difficult because the conversion probability is maximally a few percent but there are quite a lot of uncertainties in low energy atmospheric neutrinos.

\section{Acknowledgments}

This work is supported by National Science Foundation of China(NSFC), grant No.11135009, No. 11375065 and Shanghai Key Laboratory of Particle Physics and Cosmology, grant No. 11DZ2230700. 
Open Access. This article is distributed under the terms of the Creative Commons Attribution License (CC-BY 4.0), which permits any use, distribution and reproduction in any medium, provided the original author(s) and source are credited.

\section{References}

[1] P.C. de Holanda and A.Y. Smirnov, Homestake result, sterile neutrinos and low-energy solar neutrino experiments, Phys. Rev. D 69 (2004) 113002 [hep-ph/0307266] [INSPIRE].

[2] P.C. de Holanda and A.Y. Smirnov, Solar neutrino spectrum, sterile neutrinos and additional radiation in the Universe, Phys. Rev. D 83 (2011) 113011 [arXiv:1012.5627] [INSPIRE].

[3] For lower bound on $\Delta m_{01}^{2}$ we take $0.5 \times 10^{-5} \mathrm{eV}^{2}$ which comes from a discussion of figure 7 in ref. [2]. Taking the more conservative lower bound $0.7 \times 10^{-5} \mathrm{eV}^{2}$ does not change the conclusion in this article.

[4] B.T. Cleveland et al., Measurement of the solar electron neutrino flux with the Homestake chlorine detector, Astrophys. J. 496 (1998) 505 [INSPIRE].

[5] Super-Kamiokande collaboration, K. Abe et al., Solar neutrino results in Super-Kamiokande-III, Phys. Rev. D 83 (2011) 052010 [arXiv: 1010.0118] [InSPIRE].

[6] SNO collaboration, B. Aharmim et al., Low energy threshold analysis of the phase I and phase II data sets of the Sudbury Neutrino Observatory, Phys. Rev. C 81 (2010) 055504 [arXiv:0910.2984] [INSPIRE].

[7] Borexino collaboration, G. Bellini et al., Measurement of the solar $8 B$ neutrino rate with a liquid scintillator target and $3 \mathrm{MeV}$ energy threshold in the Borexino detector, Phys. Rev. D 82 (2010) 033006 [arXiv:0808.2868] [INSPIRE].

[8] L. Wolfenstein, Neutrino oscillations in Matter, Phys. Rev. D 17 (1978) 2369 [inSPIRE].

[9] L. Wolfenstein, Effect of matter on neutrino oscillations, in Neutrino-78, Purdue Univ. C3-C6 (1978).

[10] S.P. Mikheev and A.Y. Smirnov, Resonance amplification of oscillations in matter and spectroscopy of solar neutrinos, Sov. J. Nucl. Phys. 42 (1985) 913 [INSPIRE].

[11] S.P. Mikheev and A.Y. Smirnov, Resonant amplification of neutrino oscillations in matter and solar neutrino spectroscopy, Nuovo Cim. C 9 (1986) 17 [INSPIRE].

[12] S.P. Mikheyev and A.Yu. Smirnov, Neutrino oscillations in variable-density medium and Q-bursts due to gravitational collapse of stars, Sov. Phys. JETP 64 (1986) 4, reprinted in Solar neutrinos: the first thirty years, J.N. Bahcall et al. eds., Westview Press, U.S.A. (2002).

[13] H.W. Long, Y.F. Li and C. Giunti, Day-night asymmetries in active-sterile solar neutrino oscillations, JHEP 08 (2013) 056 [arXiv: 1306.4051] [INSPIRE].

[14] H.W. Long, Y.F. Li and C. Giunti, CP-violating phases in active-sterile solar neutrino oscillations, Phys. Rev. D 87 (2013) 113004 [arXiv:1304.2207] [INSPIRE].

[15] Particle Data Group collaboration, J. Beringer et al., Review of particle physics, Phys. Rev. D 86 (2012) 010001 [INSPIRE].

[16] DAYA-BAY collaboration, F.P. An et al., Observation of electron-antineutrino disappearance at Daya Bay, Phys. Rev. Lett. 108 (2012) 171803 [arXiv:1203.1669] [INSPIRE]. 
[17] RENO collaboration, J.K. Ahn et al., Observation of Reactor Electron Antineutrino Disappearance in the RENO Experiment, Phys. Rev. Lett. 108 (2012) 191802 [arXiv: 1204.0626] [INSPIRE].

[18] Daya Bay collaboration, F.P. An et al., Improved measurement of electron antineutrino disappearance at Daya Bay, Chinese Phys. C 37 (2013) 011001.

[19] E. Lisi and D. Montanino, Earth regeneration effect in solar neutrino oscillations: an analytic approach, Phys. Rev. D 56 (1997) 1792 [hep-ph/9702343] [INSPIRE].

[20] W. Liao, Precise formulation of neutrino oscillation in the Earth, Phys. Rev. D 77 (2008) 053002 [arXiv: 0710.1492] [INSPIRE].

[21] A.M. Dziewonski and D.L. Anderson, Preliminary reference Earth model, Phys. Earth. Planet. Inter. 25 (1981) 297.

[22] P.C. de Holanda, W. Liao and A.Y. Smirnov, Toward precision measurements in solar neutrinos, Nucl. Phys. B 702 (2004) 307 [hep-ph/0404042] [INSPIRE]. 